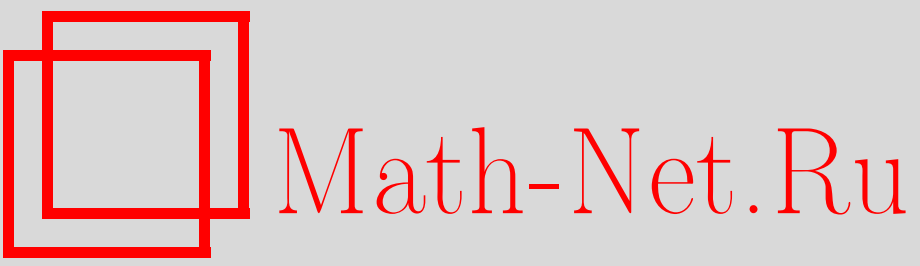

А. И. Буфетов, Эргодические теоремы для действий нескольких отображений, УМН, 1999, том 54, выпуск 4, 159-160

DOI: https://doi.org/10.4213/rm185

Использование Общероссийского математического портала Math-Net.Ru подразумевает, что вы прочитали и согласны с пользовательским соглашением

http://www.mathnet.ru/rus/agreement

Параметры загрузки:

IP: 52.23 .180 .231

26 апреля 2023 г., $07: 49: 29$ 


\title{
ЭРГОДИЧЕСКИЕ ТЕОРЕМЫ ДЛЯ ДЕЙСТВИЙ НЕСКОЛЬКИХ ОТОБРАЖЕНИЙ
}

\author{
А.И. БУфЕТОВ
}

Пусть $\left(X, B_{X}, \nu\right)$ - пространство Лебега, $f_{1}, \ldots, f_{m}: X \rightarrow X$ - измеримые отображения, сохраняющие меру $\nu$. Пусть $F_{i}$ - оператор, действующий на измеримую функцию $\varphi$ на пространстве $X$ по формуле $F_{i} \varphi=\varphi \circ f_{i}$. Обозначим через $W_{m}$ множество всех конечных слов $w=w_{1} \ldots w_{n}, w_{i}=1, \ldots, m$. Длину слова $w \in W_{m}$ обозначим через $|w|$. Для всякого $w \in W_{m}$, $w=w_{1} \ldots w_{n}$, введем оператор

$$
F_{w}=F_{w_{n}} F_{w_{n-1}} \cdots F_{w_{1}}
$$

Рассмотрим пространство $\Omega_{m}$ односторонних бесконечных последовательностей из символов $1, \ldots, m$. Для $w \in W_{m}$ пусть $C(w) \subset \Omega_{m}$ есть множество последовательностей, начинающихся словом $w$. Для произвольной меры $\mu$ на $\Omega_{m}$ обозначим $\mu(w)=\mu(C(w))$. Если $w=w_{1} \ldots w_{n}$, то вместо $\mu(w)$ будем иногда писать $\mu\left(w_{1} \ldots w_{n}\right)$.

Пусть $\mu$-стационарная марковская мера на $\Omega_{m}$. Сопоставим мере $\mu$ неориентированньй графф $\Gamma_{\mu}$ с вершинами $1, \ldots, m$, в котором вершины $i$ и $j$ соединены ребром, если найдется $l \in\{1, \ldots, m\}$ такое, что $\mu(i l)>0, \mu(j l)>0$. Меру $\mu$ будем называть сильно связной, если отвечающая ей цепь неразложима, а граф $\Gamma_{\mu}$ связен.

Далее, пусть $\mu$ - стационарная марковская мера порядка $k \geqslant 1$ на $\Omega_{m}$. Любой марковской цепи порядка $k$ с состояниями $1, \ldots, m$ стандартным образом ставится в соответствие марковская цепь порядка 1 с множеством состояний $\left\{w \in W_{m},|w|=k\right\}$. Тем самым, мере $\mu$ соответствует марковская мера $\tilde{\mu}$ порядка 1 на $\Omega_{m^{k}}$; мы называем меру $\mu$ сильно связной, если цепь, отвечающая мере $\tilde{\mu}$, неразложима, а граф $\Gamma_{\tilde{\mu}}$ имеет ровно $m^{k-1}$ компонент связности.

Всюду далее $\mu$ - стационарная марковская мера на $\Omega_{m}$ порядка $k$. Обозначим

$$
\begin{gathered}
s_{l}^{\mu}\left(F_{1}, \ldots, F_{m}\right)=\sum_{|w|=l} \mu(w) F_{w}, \\
c_{n}^{\mu}\left(F_{1}, \ldots, F_{m}\right)=\frac{1}{n} \sum_{l=0}^{n-1} s_{l}^{\mu}\left(F_{1}, \ldots, F_{m}\right) .
\end{gathered}
$$

Пусть $\varphi$-измеримая функция на $X$. Величины $c_{n}^{\mu}\left(F_{1}, \ldots, F_{m}\right) \varphi$-аналоги временны́х средних функции $\varphi$ в случае нескольких отображений. Для них имеет место аналог эргодической теоремы фон Неймана.

Teоpema 1. Пусть $\varphi \in L_{2}(X, \nu)$. Найдется функция $\bar{\varphi} \in L_{2}(X, \nu)$ maкая, что $c_{n}^{\mu}\left(F_{1}, \ldots, F_{m}\right) \varphi \rightarrow \bar{\varphi}$ в $L_{2}(X, \nu)$ при $n \rightarrow \infty$. Если мера $\mu$ сильно связна, то $F_{j} \bar{\varphi}=\bar{\varphi}$, $j=1, \ldots, m$.

Теорема 1 обобщается на случай произвольных сжатий в гильбертовом пространстве (напомним, что линейный оператор на банаховом пространстве называется сжатием, если его норма не превосходит 1). Итак, пусть $H$ - гильбертово пространство, $T_{1}, \ldots, T_{m}: H \rightarrow H$ - сжатия. Операторы $T_{w}, s_{l}^{\mu}\left(T_{1}, \ldots, T_{m}\right), c_{n}^{\mu}\left(T_{1}, \ldots, T_{m}\right)$ определяются по аналогии с (1), (2) и (3).

Tеорема 2. Для всякого $h \in H$ найдется вектор $\bar{h}$ maкой, что $c_{n}^{\mu}\left(T_{1}, \ldots, T_{m}\right) h \rightarrow \bar{h}$ в $Н$ при $n \rightarrow \infty$. Если мера $\mu$ сильно связна, то $\mathrm{T}_{j} \bar{\varphi}=\bar{\varphi}, j=1, \ldots, m$.

Для средних (3) верна также индивидуальная эргодическая теорема. Мы ограничиваемся здесь случаем пространства конечной меры.

Работа частично поддержана грантами РФФИ 98-01-00455 и INTAS 93-570-ext. 
Teорема 3. Пусть $\nu(X)<\infty, \varphi \in L_{1}(X, \nu)$. Найдется функция $\bar{\varphi} \in L_{1}(X, \nu)$ такая, что $c_{n}^{\mu}\left(F_{1}, \ldots, F_{m}\right) \varphi \rightarrow \bar{\varphi} \quad \nu$-почти всюду и в $L_{1}(X, \nu) \quad$ при $n \rightarrow \infty$. При этом $\int_{X} \varphi=\int_{X} \bar{\varphi}$.

Если мера $\mu$ сильно связна, то $F_{j} \bar{\varphi}=\bar{\varphi}, j=1, \ldots, m$.

Пусть $I_{X}-\sigma$-алгебра $f_{1}, \ldots, f_{m}$-инвариантных подмножеств $X$. Из теоремы 3 видно, что если мера $\mu$ сильно связна, то $\bar{\varphi}=\mathrm{E}\left(\varphi \mid I_{X}\right)$ - условное математическое ожидание функции $\varphi$ относительно $\sigma$-алгебры $I_{X}$.

Теорема 3 обобщается на случай произвольных положительных $L_{1}-L_{\infty}$-сжатий.

Tеорема 4. Пусть $\nu(X)<\infty, T_{1}, \ldots, T_{m}: L_{1}(X, \nu) \rightarrow L_{1}(X, \nu)$ - положительные $L_{1}-L_{\infty}$-сжатия. Пусть $\varphi \in L_{1}(X, \nu)$. Найдется функция $\bar{\varphi} \in L_{1}(X, \nu)$ такая, что $c_{n}^{\mu}\left(T_{1}, \ldots, T_{m}\right) \varphi \rightarrow \bar{\varphi} \nu$-почти всюду и в $L_{1}(X, \nu)$ при $n \rightarrow \infty$.

Если мера н сильно связна, то $T_{j} \bar{\varphi}=\bar{\varphi}, j=1, \ldots, m$.

Теоремы 1, 3 обобщают эргодические теоремы для действий свободных групп, полученные Р.И. Григорчуком [1], [2] и независимо А. Нево и Э. М. Стайном [3], а также эргодические теоремы для действий свободных полугрупп, принадлежащие Р. И. Григорчуку [2]. Чтобы получить теоремы Григорчука для действий свободных полугрупп сохраняющими меру отображениями, возьмем в качестве $\mu$ меру Бернулли. Чтобы получить теоремы из [1]-[3] для действия свободной группы с $l$ образующими, положим $m=2 l$, пусть $g_{1}, \ldots, g_{l}$-образующие в группе, $g_{l+i}=g_{i}^{-1}$, а сильно связная мера Маркова порядка 1 задается матрицей переходных вероятностей $\left(a_{i j}\right)$, $i, j=1, \ldots, m$, где $a_{i, i+l}=a_{i+l, i}=0$ при $1 \leqslant l, a_{i j}=1 /(m-1)$ при $|i-j| \neq l$ (иншми словами, запрещены переходы из образующего в его обратньй и, наоборот, все прочие переходы равновероятны).

Теоремы 1, 3 могут также быть использованы для получения эргодических теорем для действий полугрупп с соотношениями. Полугруппу $G$ с образующими $g_{1}, \ldots, g_{m}$ будем называть $n o$ лугруппой конечного типа, если найдется такой набор запретных слов в алфавите $g_{1}, \ldots, g_{m}$, что всякий элемент полугруппы однозначно записывается словом, не содержащим запретных. Для полугрупп конечного типа можно так выбрать меру $\mu$, что каждый элемент полугруппы будет учтен не более одного раза в эргодических средних (3), и теоремы 1,3 дадут тогда эргодические теоремы для действий таких полугрупп.

Я глубоко благодарен Д.В. Аносову, Р.И. Григорчуку, Б.М. Гуревичу, Ю. С. Ильяшенко, В. И. Оселедщу, В.А. Тиморину и А.Ю. Фишкину за полезные обсуждения.

\section{СПИСОК ЛИТЕРАТУРЫ}

[1] Григорчук Р. И. Индивидуальная эргодическая теорема для действий свободных групп // Тезисы Тамбовской школы по теории функций, 1986. [2] Григорчук Р. И. // Матем. заметки. 1999. Т. 65. № 5. С. 779-783. [3] Nevo A., Stein E. M. // Acta Math. 1994. V. 173. P. $135-154$. 